\title{
An adaptive Sliding Window based on Fuzzy Filter for removing Wide-range Impulse Noise Densities on the Image Sequence
}

\author{
Fitri Utaminingrum $^{\mathrm{a}, \mathrm{b}, \text { * }}$, Keiichi Uchimura ${ }^{\mathrm{a}}$, Gou koutaki ${ }^{\mathrm{a}}$ \\ ${ }^{a}$ Kumamoto University, 2-39-1 Kurokami Kumamoto, 860-8555, Japan \\ ${ }^{\mathrm{b} B r a w i j a y a}$ University, Veteran No. 8 Malang, 65145, Indonesia \\ *Corresponding Author: f3-ningrum@navi.cs.kumamoto-u.ac.jp
}

\begin{abstract}
In this work, we propose a filter using adaptive sliding window based on a fuzzy filter to reduce impulse noise corruption in the image sequence. Two uncorrupted pixels are selected from $w_{t}$ and $w_{t+1}$ windows. An effective method for reducing of impulse noise and obtaining a fast computation time process is presented in our research paper by adopting a fuzzy theory. Fuzzy is knowledge-based and robust, that cause several methods based on fuzzy method will get a better result in the filtering image. Experimental results of quantitative and qualitative parameters have provided a high Mean Structural Similarity (MSSIM) index, a high Peak Signal to Noise Ratio (PSNR), a good visual result and a fast computing time at various percentages of impulse noise densities in the image sequence.
\end{abstract}

Keywords: window, impulse noise, pixel.

\section{Introduction}

Image sequence processing is a field that continues to grow, with new applications being developed in many aspects. Image sequence processing usually refers to a two-dimensional digital signal processing by using a computer. Displaying several frames of two-dimensional images in the sequence image creates the illusion of motion.

The image sequences are likely to be corrupted by noise due to bad acquisition in transmission or in the recording process ${ }^{(1,2,3)}$. The noise can be visible as the stains of noise or the disturbance pixel.

The denoising process is always accompanied by the loss of image details ${ }^{(4)}$. One type of the disturbance in the digital image is impulse noise. Impulse noise is short-duration noise that occurs in the digital image due to errors in the switching, interference from the channel or due to atmospheric disturbances during image transmission ${ }^{(5)}$. Impulse noise is modeled as several bit errors in the signal values during transmitting the images or video sequences over noisy data links ${ }^{(6)}$.

For removing impulse noise in the image sequence is needed the filtering process. The filtering process in the image sequence refers to a set of digital image related to a noise removal contents.

Many impulse noises-removal techniques have been developed to suppress impulse noise while preserving image details ${ }^{(7,8,9)}$. Median filter is the most popular kind of nonlinear filter that has been extensively used for removing of impulse noise due to its simplicity ${ }^{(10)}$. Median filters are known to remove impulse noise and preserve edges. However, a standard median filter has a low capability to reduce the artifacts of the highly corrupted image. The median filter still blurry in some fine details and often damages edges when it is applied to the digital images uniformly ${ }^{(11)}$.

Lone Diagonal Sorting (LDS) algorithm uses diagonal sorting direction, which applied in the current frames ${ }^{(12)}$. The sorting process is only conducted on the diagonal part of the sample pixel window. Because of the analysis is only on the diagonal direction which causes the quality of the filtering result not well enough.

The output of non-recursive filter structure depends only on the input. It has no feedback paths. Meanwhile, the output recursive filter structures depend both on the input and on the previous output. It has feedback paths. Recursive implementations also require smaller memory space than non-recursive implementation ${ }^{(13)}$. Recursive filters are an 


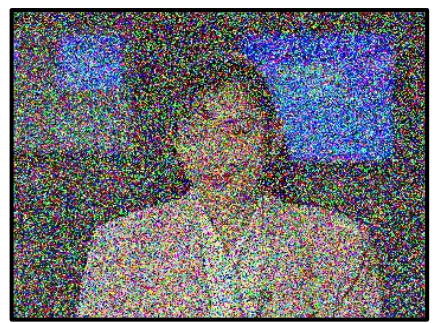

$f(x, y, t-2)$

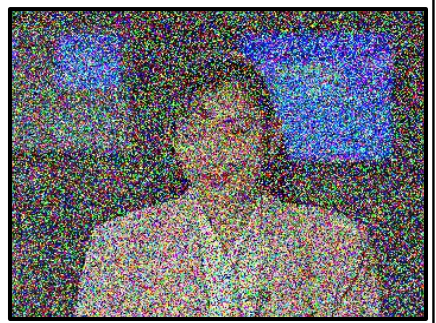

$f(x, y, t-1)$

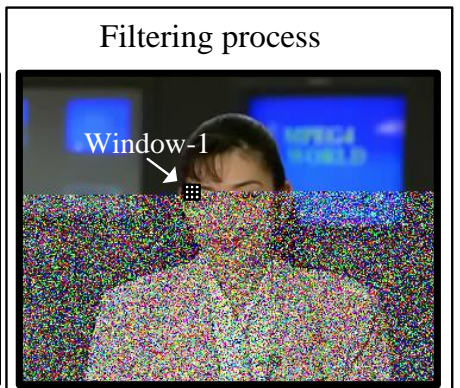

$f(x, y, t)$

Current frame
The frame has been filtered

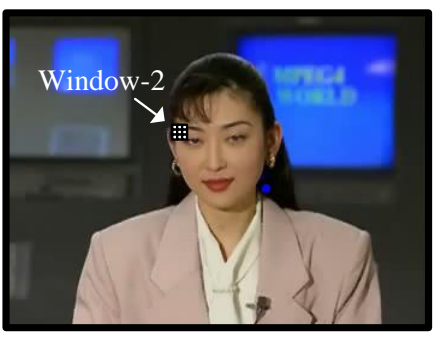

$f(x, y, t+1)$

Fig. 1. Illustration of the corrupted image sequence filters.

efficient way to remove impulse noise. However, they have less performance and flexibility than other digital filters. Recursive implementations also require smaller memory space than non-recursive implementation ${ }^{(13)}$.

We adopt a fuzzy in our research, It is knowledge-based and robust, that cause several methods based on fuzzy sets will get a better result in the image processing $^{(14,15)}$.

In order to overcome the drawback of the previous research, an effective method for reducing of impulse noise and obtaining a fast computation time process is proposed in our research paper by adopting a fuzzy theory.

The rest of this paper is organized as follows. Explanation of our proposed method is presented in the Section 2. The simulation result is discussed in Section 3. Finally, the conclusion of our research is presented in Section 4.

\section{Proposed Method}

In this paper, we propose a filter using adaptive sliding window based on fuzzy filter. The sliding window is illustrated in $f(x, y, t)$ and $f(x, y, t+1)$ frames as shown in Fig.1. Meanwhile, the filtering process is presented in Fig. 2. A sample pixel window in the $f(x, y, t)$ frame is symbolized by $w_{t}$, whereas for the $f(x, y, t+l)$ frame is symbolized $w_{t+1}$. Two uncorrupted pixels $\left(P_{1}\right.$ and $\left.P_{2}\right)$ are selected from $w_{t}$ and $w_{t+1}$. Furthermore, the weight values of $p_{1}$ and $p_{2}$ are obtained by using a fuzzy method. The selection process of two pixels is described in the subsection 2.1.

Description of the corrupted image sequence filters starting from left to the right side on Fig.1 is divided into three parts. The first part is $f(x, y, t-1)$ and $f(x, y, t-2)$ frames as the corrupted pixel that will be filtered. The second part is $f(x, y, t)$ as the filtering process in the current frame. The last part on the right-side of Fig. 1 is the frame that has been filtered.

\subsection{Sample Pixel Window}

Sample pixel window $w_{t}$ and $w_{t+1}$ are successively taken from the current frame of $f(x, y, t)$ and $f(x, y, t+1)$. Matrix size for the $w_{t}$ and $w_{t+1}$ has the similar size. Both are $3 \times 3$. Both of matrices can be written in the mathematical formulation as shown in Eqs.1 and 2. The $w_{t}$ and $w_{t+1}$ windows shift together at the similar coordinates for each frame.

$$
\begin{aligned}
& w_{t}=\left|\begin{array}{ccc}
f(x-1, y-1, t) & f(x-1, y, t) & f(x-1, y+1, t) \\
f(x, y-1, t) & f(x, y, t) & f(x, y+1, t) \\
f(x+1, y-1, t) & f(x+1, y, t) & f(x+1, y+1, t)
\end{array}\right| \\
& w_{t+1}=\left|\begin{array}{ccc}
f(x-1, y-1, t+1) & f(x-1, y, t+1) & f(x-1, y+1, t+1) \\
f(x, y-1, t+1) & f(x, y, t+1) & f(x, y+1, t+1) \\
f(x+1, y-1, t+1) & f(x+1, y, t+1) & f(x+1, y+1, t+1)
\end{array}\right|
\end{aligned}
$$

\subsection{Sample Pixel Window}

Two uncorrupted pixels are selected from $w_{t}$ and $w_{t+1}$ windows. The first, two pixels are searched in the $w_{t}$ frame sequences starting from the Horizontal direction $(\mathrm{H})$, Vertical direction (V), Left Diagonal (LD) and Right Diagonal (RD), as shown in Fig.3. Two pixels in the $w_{t}$ window are taken only if the position of the uncorrupted pixel direction as illustrated in Fig.3. The second, if two pixels are not found in the $w_{t}$ window, then continued by combining of $w_{t}$ and $w_{t+1}$ windows are shown in Fig.4. Figure 4 shows a combination of the two pixels election. One pixel is taken from $w_{t}$ and one other pixel from $w_{t+1}$. The similar way is also applied to another direction. 


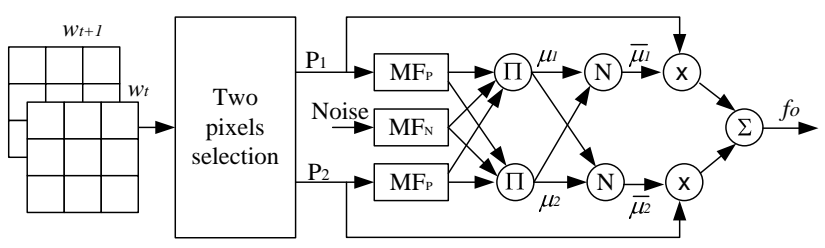

Fig. 2. The filtering process
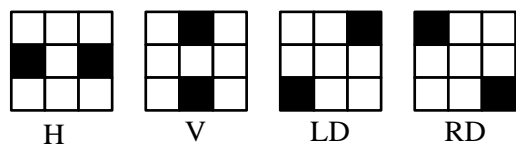

Fig. 3. Two uncorrupted pixels from windows $w_{t}$ and $w_{t+1}$

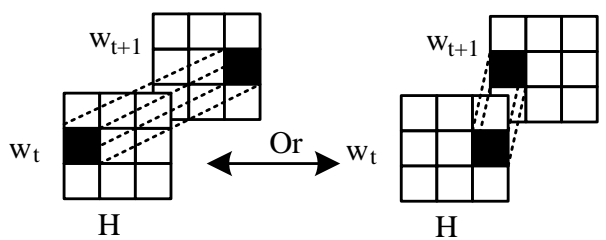

Fig. 4. Pixel selection direction

\section{$2.3 \quad$ Fuzzy}

We use two Membership Functions. Membership Function (MF) is used to the pixel that symbolized by $\left(\mathrm{MF}_{\mathrm{P}}\right)$, and for noise symbolized by $\left(\mathrm{MF}_{\mathrm{N}}\right)$. Meanwhile, $\mathrm{MF}$ for the output is similar with MF for pixel. Those have five memberships. $\mathrm{MF}_{\mathrm{P}}$ for $P_{1}$ and $P_{2}$ are illustrated in Fig.2 that is obtained from the graph of MF as shown in Fig.5.

Membership from the pixel intensity $(0-255)$ is divided into five among others are Low (L), Low Medium (LM), Medium (M), Medium High (MH) and High $(\mathrm{H})$. The membership function of pixel intensity uses $\mathrm{MF}$ triangle.

$\mu$ is the membership degree of MF. Furthermore, MF for noise (MFN) is shown in Fig.6. Membership degree $(\mu)$ value can be calculated using Eqs.(3) and (4).

$$
\begin{aligned}
& \mu_{1}=\frac{x_{1}-a_{1}}{b_{1}-a_{1}} \\
& \mu_{2}=\frac{b_{2}-x_{2}}{b_{2}-a_{2}}
\end{aligned}
$$

Equation 3 is used to uphill curve while Eq.(4) for downhill curve. Furthermore, uphill curve and downhill curve are shown in Figs.7 and 8 successively. There are ten rules to obtain variable output fuzzy $\left(f_{o}\right)$ that are used in the proposed filter. A rule is a combination of input $P_{1}, P_{2}$ and noise. Ten fuzzy rules consist of:

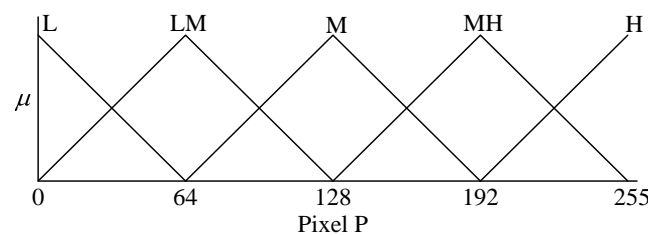

Fig. 5. Membership function for pixel $\left(\mathrm{MF}_{\mathrm{P}}\right)$

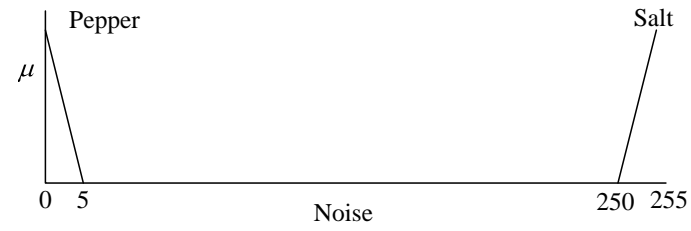

Fig. 6. Membership function for Noise $\left(\mathrm{MF}_{\mathrm{N}}\right)$

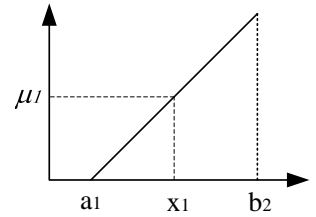

Fig. 7. Uphill curve

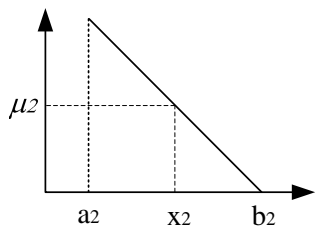

Fig. 8. Downhill curve

1. If $P_{1}$ is $\mathrm{L}$ and $P_{2}$ is $\mathrm{LM}$ and noise is Salt then $f_{o}$ is $\mathrm{L}$.

2. If $P_{1}$ is $\mathrm{LM}$ and $P_{2}$ is $\mathrm{L}$ and noise is Salt then $f o$ is $\mathrm{L}$.

3. If $P_{1}$ is LM and $P_{2}$ is $\mathrm{M}$ and noise is Salt then fo is LM.

4. If $P_{l}$ is $\mathrm{M}$ and $P_{2}$ is LM and noise is Salt then $f_{o}$ is LM.

5. If $P_{1}$ is $\mathrm{M}$ and $P_{2}$ is $\mathrm{M}$ and noise is Salt then $f o$ is $\mathrm{M}$.

6. If $P_{1}$ is $\mathrm{M}$ and $P_{2}$ is $\mathrm{MH}$ and noise is pepper then $f_{o}$ is M.

7. If $P_{1}$ is $\mathrm{MH}$ and $P_{2}$ is $\mathrm{M}$ and noise is pepper then $f o$ is $\mathrm{M}$.

8. If $P_{1}$ is $\mathrm{MH}$ and $P_{2}$ is $\mathrm{MH}$ and noise is pepper then fo is $\mathrm{MH}$.

9. If $P_{1}$ is $\mathrm{MH}$ and $P_{2}$ is $\mathrm{H}$ and noise is pepper then $f o$ is $\mathrm{MH}$.

10. If $P_{1}$ is $\mathrm{H}$ and $P_{2}$ is $\mathrm{MH}$ and noise is pepper then $f o$ is $\mathrm{MH}$.

The total of membership degrees $\left(\mu_{1}\right.$ and $\left.\mu_{2}\right)$ should be equal to 1 . It is intended that the pixel data value does not exceed from maximum intensity (255). Hence, normalization of $\mu_{1}$ and $\mu_{2}$ performed by using Eqs.(5) and (6). 


$$
\begin{aligned}
& \bar{\mu}_{1}=\frac{\mu_{1}}{\mu_{1}+\mu_{2}} \\
& \bar{\mu}_{2}=\frac{\mu_{2}}{\mu_{1}+\mu_{2}}
\end{aligned}
$$

After $\bar{\mu}_{1}$ and $\bar{\mu}_{2}$ was obtained, the output value of the fuzzy can be calculated by summing from multiplication of each pixel with membership degree values, as written in equation (7).

$$
f o=P_{1} \overline{\mu_{1}}+P_{2} \overline{\mu_{2}}
$$

\section{Simulation Result}

Akiyo and xylophone image sequences have been used to test the performance of filtering results in the several methods. Images will be corrupted by salt-and-pepper noise at different noise densities, start from low noise (10\%) up to high noise (90\%). This paper presents a set of qualitative and quantitative scores designed to assess performance of any filtering methods.

\subsection{Qualitative Parameter}

Qualitative measurements are ways of collecting data, which are concerned with visual observation of the filtering results. The numerous advantages of qualitative methods provide a depth of understanding of visual quality of the filtering performance process. The qualitative investigation is more informative as shown in Figs. 9 to 12. Figures 9 and 11 are the sample frames visual result of the filtering process in several methods to reduce a high impulse noise density (50\% and 90\%), respectively.

Figure 9(a) is the corrupted image of impulse noise density $50 \%$, original information about Akiyo image is still visible, although the image quality is very low. Adaptive median filtering results are still contained much noise as shown in Fig.9(b). Fig 9(c) is LDS filtering results. It still looks noise and there are certain parts have a damaged area such as Akiyo hair, television, etc. Figures 9(d) and (e) are the filtering results of non-recursive and recursive, respectively. Both methods have a rough texture. Fig 9(f) is the filtering result of the proposed method.

Figure 12(a) is the corrupted image of impulse noise density $90 \%$, original information about xylophone image is not visible. Adaptive median filtering results are still contained much noise. The image appears spots that tend white color as shown in Fig.12(b). Figure 12(c) is LDS filtering results. LDS method has a better quality than
Adaptive Median. However, the image quality is still low. The filtering results of non-recursive and recursive as shown in Figs.12(d) and (e) have rough texture. Both methods have an impulse noise with black dominant color. Fig 9(f) is the filtering result of the proposed method. Our method is better than Figs.(a), (b), (c), (d) and (e).

Meanwhile, Figures.10 and 12 are SSIM map that is used to analysis performance of the filtering result. SSIM index is calculated within the local window ${ }^{(15)}$. The higher illumination of the SSIM index maps, the quality image of the filtering result is more similar to the original image and vice versa. SSIM map of our method has a higher illumination color.

Figure 10(a) is SSIM map of the Akiyo corrupted image $(50 \%)$. It has a black dominant color in all image area. SSIM map of adaptive median method has a lower illumination as shown in Fig.10(b). Meanwhile, LDS method in Fig.10(c) has SSIM map is better than Adaptive Median Filter. Figures 10(d) and (e) are SSIM maps from non-recursive and recursive methods. Those have the illumination quality almost similar with an Adaptive Median method. SSIM map of our method in Fig 10(f) has a higher illumination than Figs.10(a), (b), (c), (d) and (e).

Furthermore, Fig.12(a) is SSIM map of the Xylophone corrupted image (90\%) has a black color in all image area. SSIM map of Adaptive Median method in Fig.12(b) has a black dominant color. Meanwhile, SSIM map of LDS method in a higher impulse noise as shown in Fig.12(c) also has black dominant color. However, edge texture of xylophone image is a little visible. Figures 12(d) and (e) are SSIM maps from non-recursive and recursive methods. Both methods have the illumination quality almost similar with an Adaptive Median method. Meanwhile, SSIM map of our method in Fig 12(f) has a higher illumination than Figs.12(a), (b), (c), (d) and (e). SSIM map of our method

\subsection{Quantitative Parameter}

The quantitative analysis can give more precise and objective information about the filtering result using the numeric data evaluation. The quantitative parameter measurements used to compare the restoration performance of various filters were the Peak Signal-to-Noise Ratio (PSNR) and Mean Structural Similarity (MSSIM) index for measuring the similarity between two images. In addition, the computing time is also used for calculating the length from the computation time of the filtering process. 


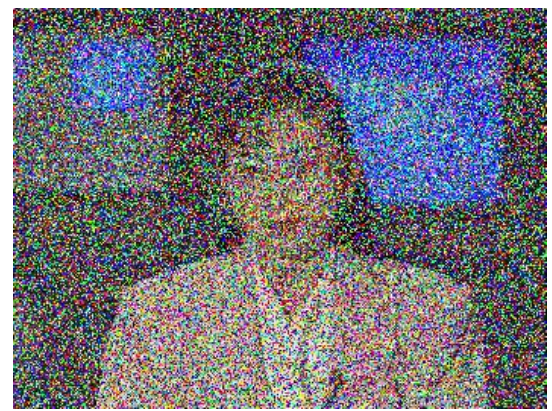

(a) Corrupted image

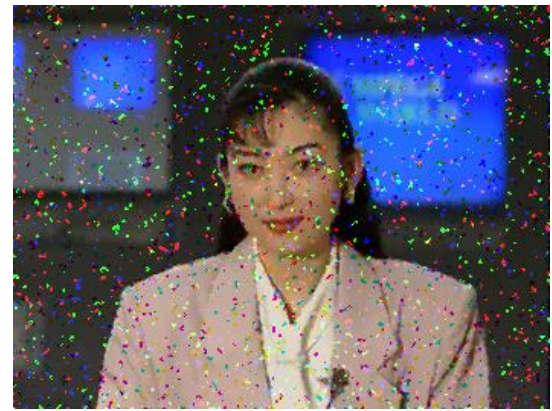

(b) Adaptive Median

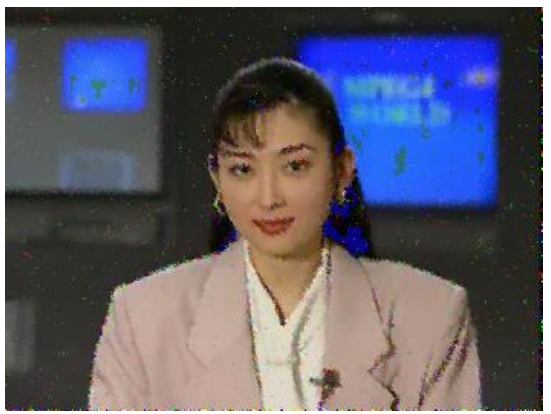

(c) LDS

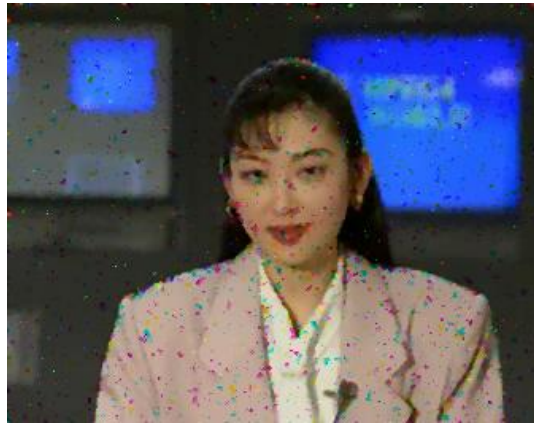

(d) Non Recursive

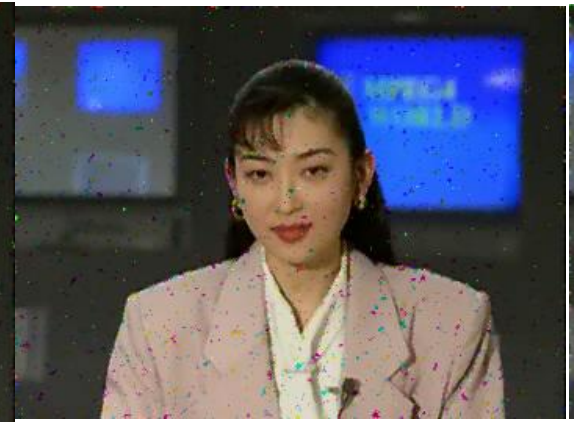

(e) Recursive

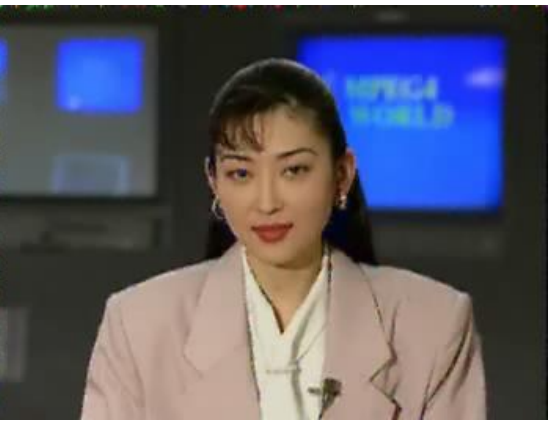

(f) Our method

Fig. 9. Filtering results for Akiyo corrupted image $50 \%$

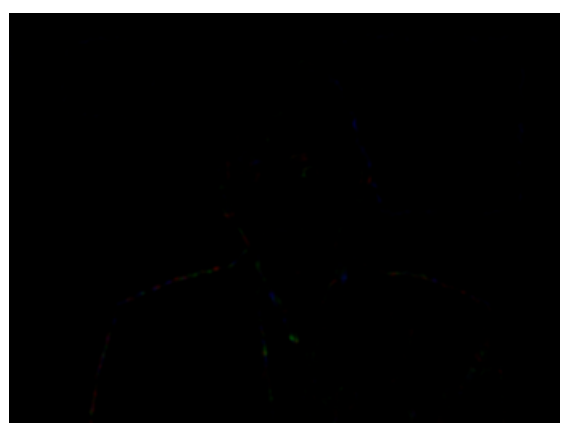

(a) Corrupted image

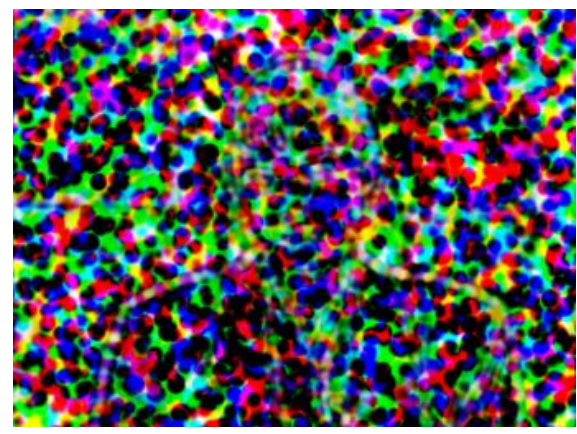

(d) Non Recursive

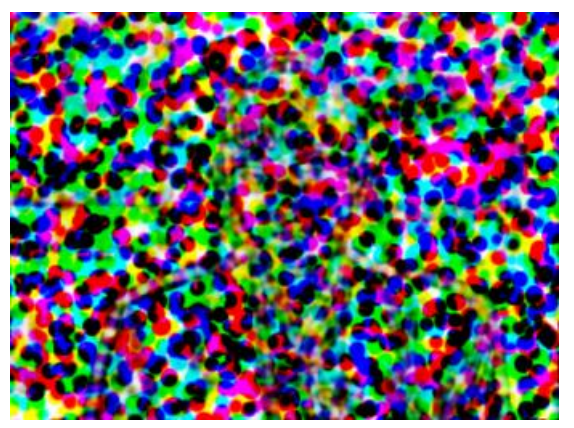

(a) Adaptive Median

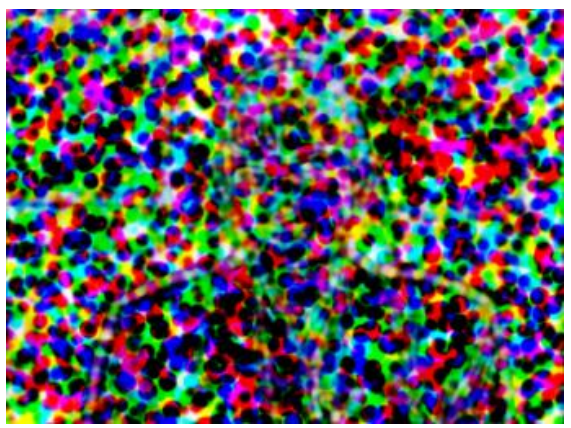

(e) Recursive

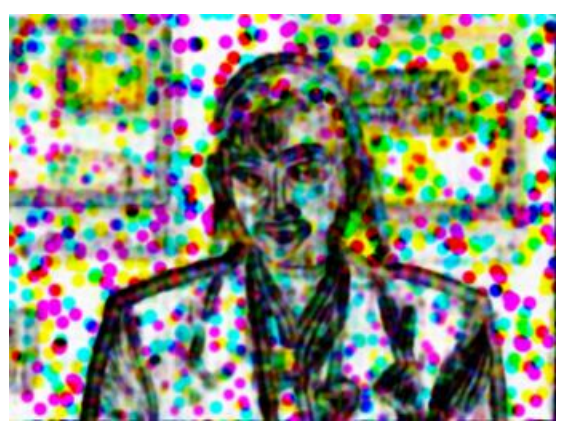

(c) LDS

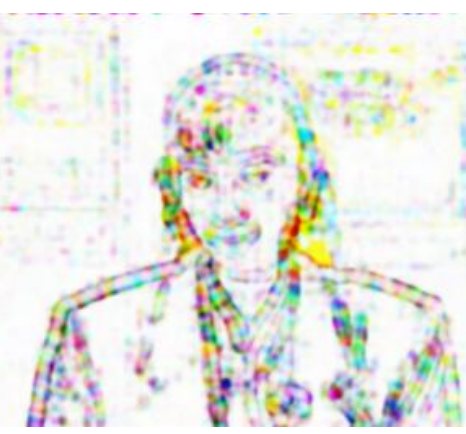

(f) Our method

Fig. 10. SSIM map for Akiyo image is used to analysis impulse noise 50\% 


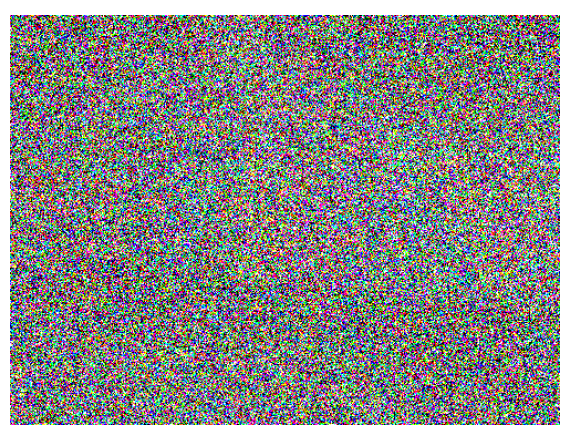

(a) Corrupted image

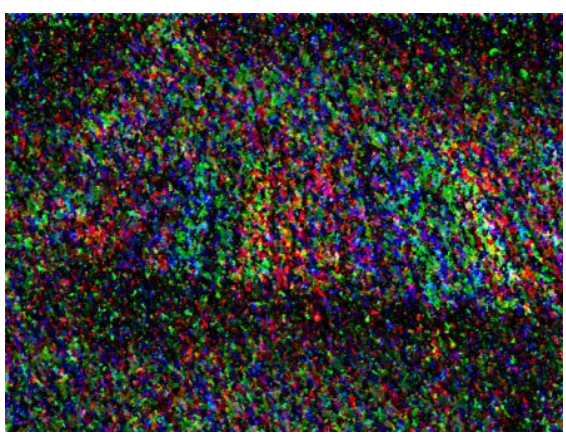

(d) Non Recursive

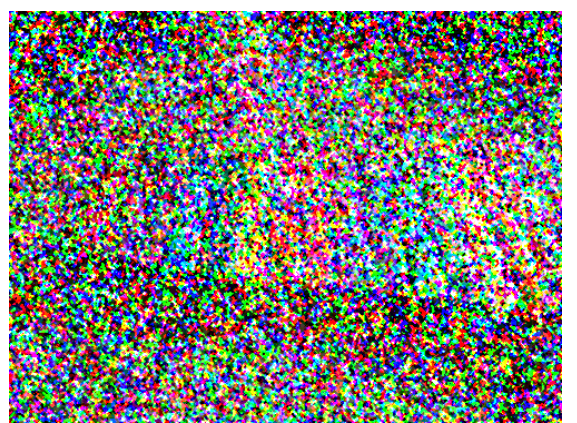

(b) Adaptive Median

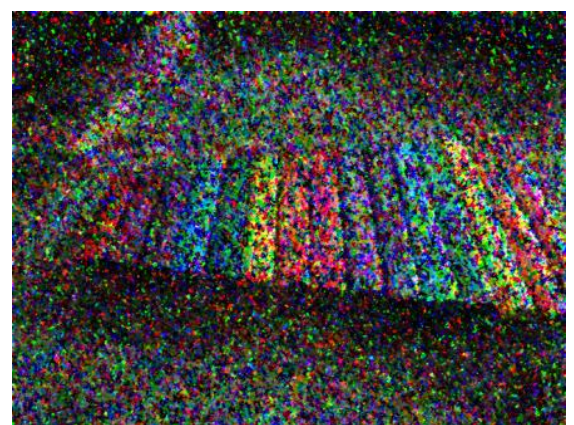

(e) Recursive

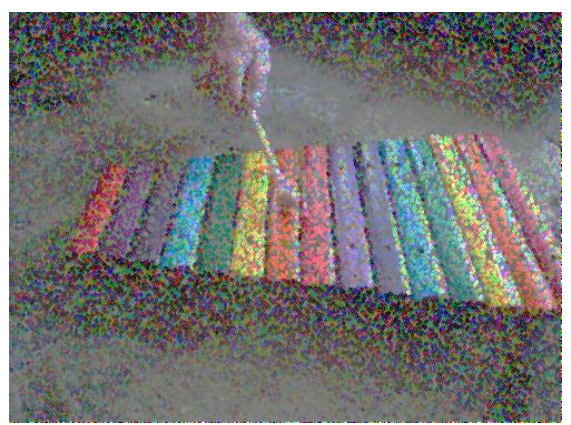

(c) LDS

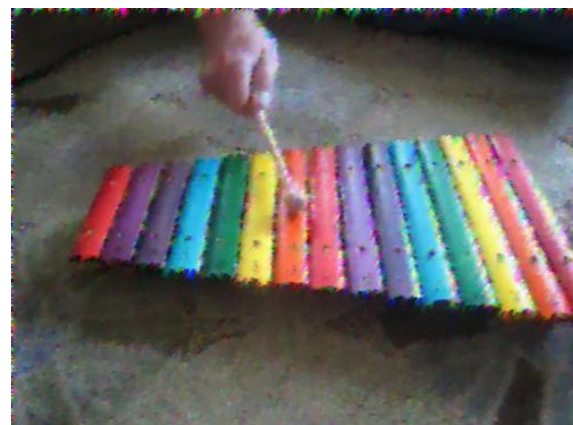

(f) Our method

Fig. 11. Filtering results for Xylophone corrupted image $90 \%$

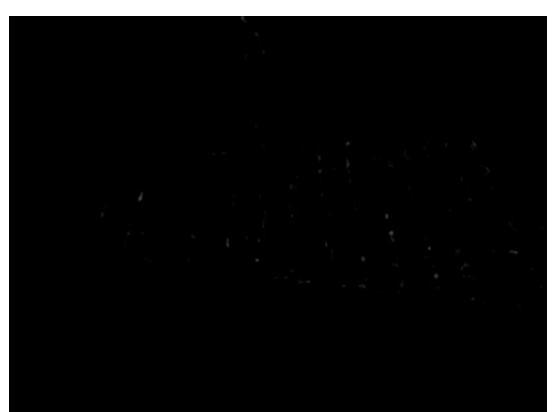

(a) Corrupted image

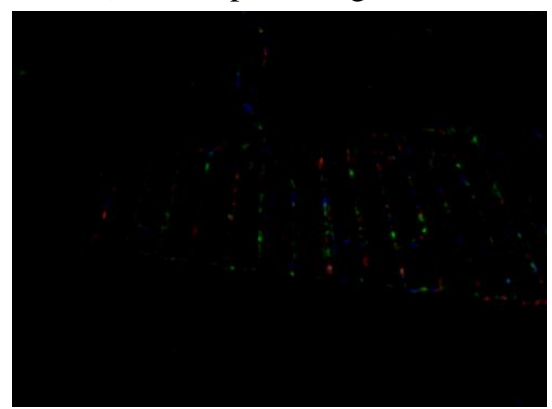

(d) Non Recursive

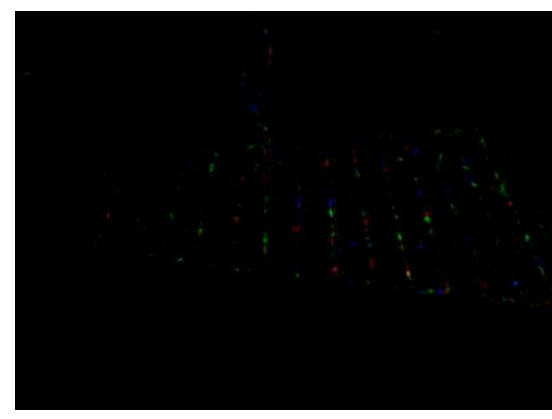

(b) Adaptive Median

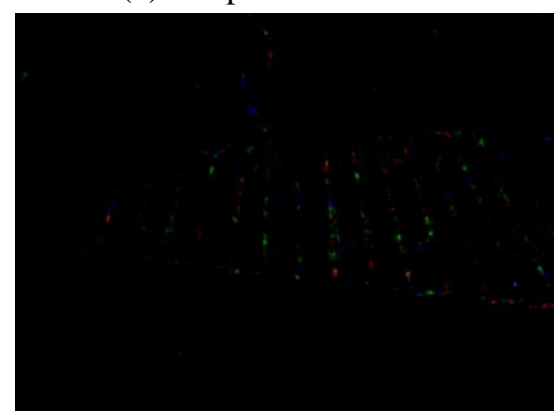

(e) Recursive

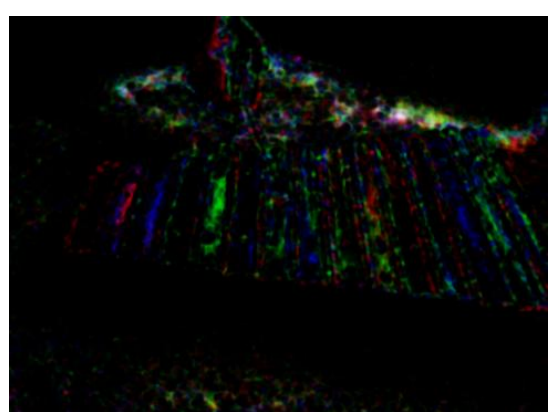

(c) $\operatorname{LDS}$

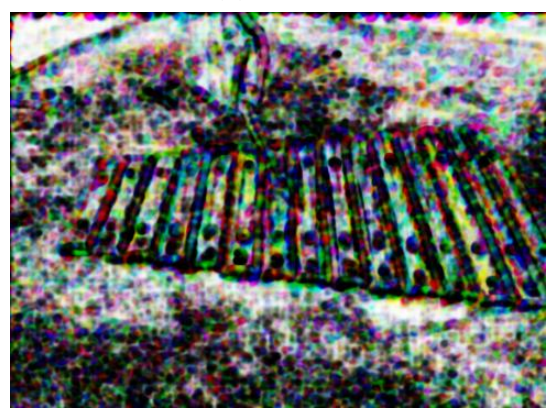

(f) Our method

Fig. 12. SSIM map for Xylophone image is used to analysis impulse noise $90 \%$ 


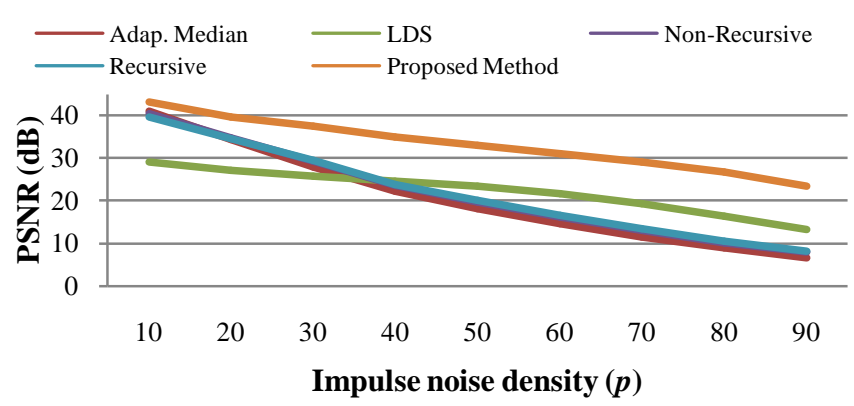

Fig. 13. PSNR Akiyo image

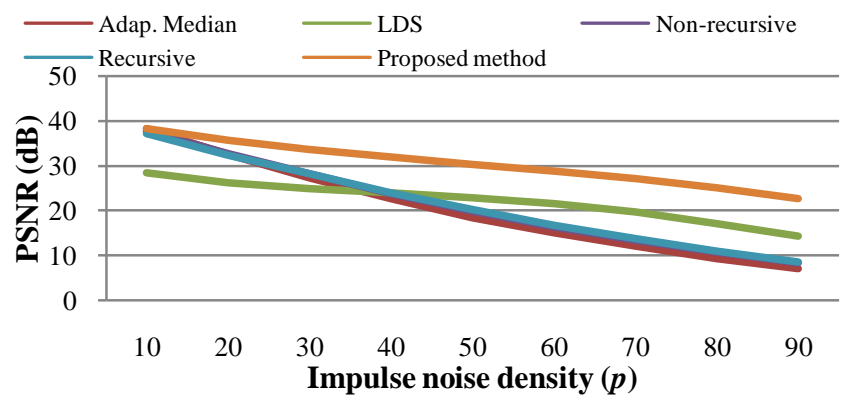

Fig. 14. PSNR Xylophone image

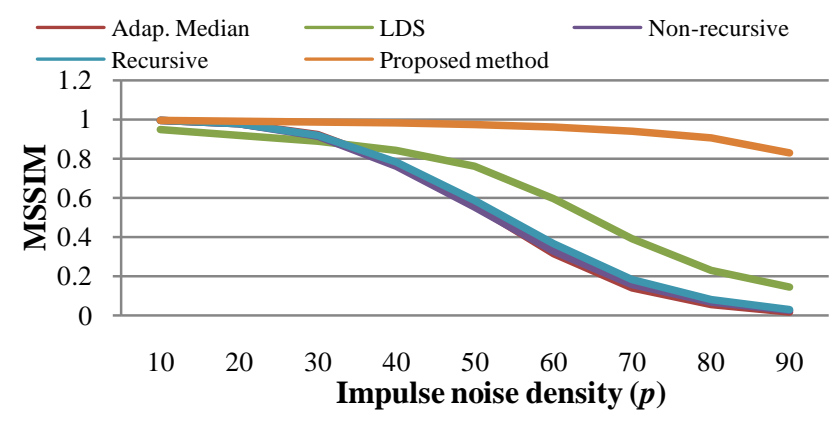

Fig. 15. MSSIM Akiyo image

PSNR parameter is often used as a benchmark level of similarity between the final filtering image result that has been reconstructed $\left(f^{\prime}(x, y)\right)$ and the reference image $(f(x, y))$. Equation (8) is used to calculate PSNR result of the filtering results. $M$ is column and $N$ is row of the image. The bigger PSNR value, the better image quality and vice versa.

$$
\text { PSNR }=10 \log _{10} \frac{255^{2} \mathrm{M} . \mathrm{N}}{\sum_{\mathrm{y}=1}^{\mathrm{M}} \sum_{\mathrm{x}=1}^{\mathrm{N}}\left(\mathrm{f}(\mathrm{x}, \mathrm{y})-\mathrm{f}^{\prime}(\mathrm{x}, \mathrm{y})\right)^{2}}
$$

Figures 13 and 14 are PSNR values of Akiyo and Xylophone image, respectively. Referring to Figs.13 and 14, our proposed methods at small impulse noise (10\%) almost have similar PSNR value with recursive methods. However, PSNR values always have a higher value than Adaptive Median, LDS, Non-recursive and recursive methods at the impulse noise densities more than $10 \%$.

Meanwhile, MMSIM index is calculated by using Eq.(9). Where, $x$ and $y$ are the reference image and the

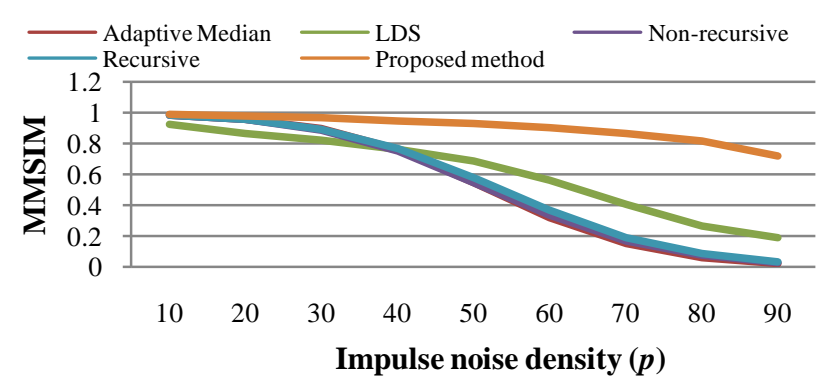

Fig. 16. MSSIM Xylophone image

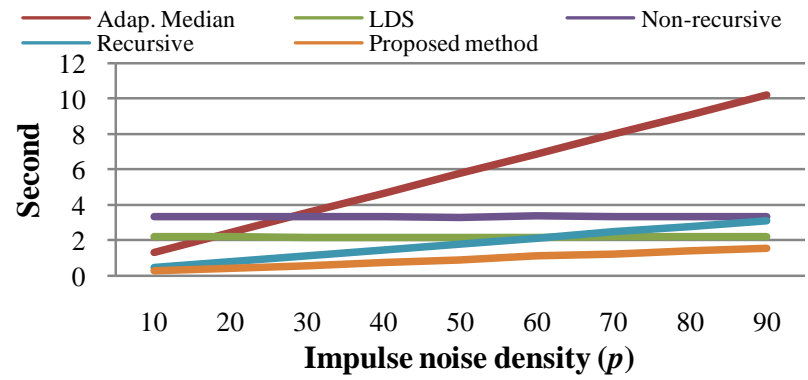

Fig. 17. Computation time Akiyo image

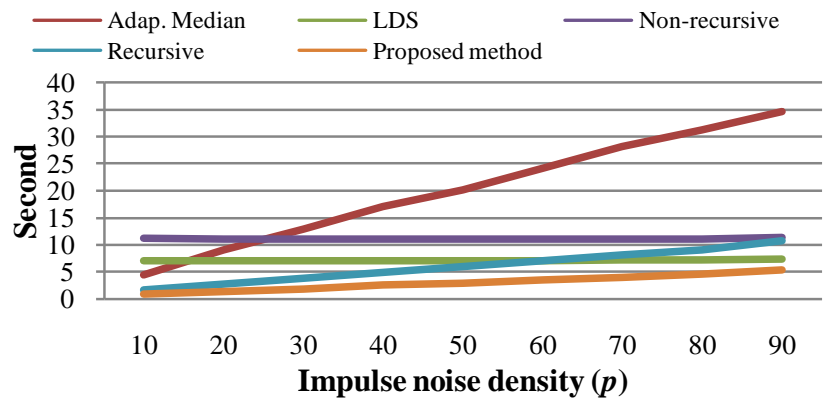

Fig. 18. Computation time Xylophone image

distorted images, respectively. $x_{j}$ an $y_{j}$ are the image contents at the local window. Furthermore, $M$ is the number of local windows in the image ${ }^{(16)}$.

$$
\operatorname{MSSIM}=\frac{1}{M} \sum_{j=1}^{M} \operatorname{SSIM}\left(x_{j}, y_{j}\right)
$$

MSSIM index values of Akiyo and Xylophone are presented in Figs. 15 and 16, respectively. Figures 15 and 16 have SSIM index value almost similar to the recursive method in the impulse noise density $10 \%$ and $20 \%$. Furthermore, SSIM index has higher value than Adaptive Median, LDS, Non-recursive and recursive methods at impulse noise densities more than $20 \%$.

Furthermore, computation time results of Akiyo and Xylophone in our proposed method in the impulse noise $10 \%$ is a little faster than Recursive method as shown in Figs.17 and 18. While, our proposed method is seen more quickly in the computation time process than all comparison methods at impulse noise densities more than $20 \%$ up to $90 \%$. 


\section{Conclusions}

Generally, the complex image processing requires a long time in the computing time to optimize the filtering result. Therefore, it is important to obtain a good visual result and a fast computation time in the filtering process. Adaptive sliding window based on a fuzzy filter to reduce impulse noise corruption in the image sequence is proposed. An effective method for reducing an impulse noise and obtaining a fast computation time process is obtained by reducing input fuzzy to become two inputs $\left(P_{1}\right.$ and $\left.P_{2}\right)$. Experimental results of quantitative and qualitative parameters have a good result.

For future work, we have plans to create Universal filter by combining the capability of our proposed method with Gaussian filter.

\section{Acknowledgment}

The first author would like to thank the Directorate General of Higher Education Ministry of National Education (DGHE) of Indonesia which providing scholarship.

\section{References}

(1) Tom Mélange, Mike Nachtegael, Stefan Schulte and Etienne Kerre : "A fuzzy filter for the removal of random impulse noise in image sequences", Image and Vision Computing, Vol. 29, Issue. 6, pp. 407-419, 2011.

(2) Xuming Zhang, Yi Zhan, Mingyue Ding, Wenguang Hou and Zhouping Yin : "Decision-based non-local means filter for removing impulse noise from digital images", Signal Processing, Vol. 93, Issue. 2, pp. 517-524, 2013.

(3) H.L. Eng, K.K. Ma : "Noise adaptive soft-switching median filter", IEEE Transactions on Image Processing Transactions, Vol.10, No.2, pp. 242-251, 2001.

(4) Ali. S. Awad : "Localizing and restoring clusters of impulse noise based on the dissimilarity among the image pixels", Journal of Advances in Signal Processing, 161, pp. 1-7, 2012.

(5) Lakshmanan. S, Mythhili. C and Kavitha.V : "A Different Cameras Image Impulse Noise Removal Technique", International Journal on Computer Science and Engineering (IJCSE), Vol. 4 No. 06, pp. 1030-1034, 2012.
(6) Gouchol Pok, Jyh-Charn Liu, and Attoor Sanju Nair : "Selective removal of impulse noise based on homogeneity level information", IEEE Transactions on Image Processing, Vol. 12, No.1, pp. 85-92, 2003.

(7) J. Harikiran, B. Saichandana and B. Divakar : "Impulse Noise Removal in Digital Images", International Journal of Computer Applications, Vol. 10, No.8, pp. 39-42, 2010.

(8) Tzu-Chao Lin and Pao-Ta Yu : "Adaptive two-pass median filter based on support vector machine for image restoration", Journal of Neural Computation, Vol. 16, No.2, pp. 333-354, 2004.

(9) Tao Chen and Hong Ren Wu : "Application of partition-based median type filters for suppressing noise in images", IEEE Transactions on Image Processing, Vol. 10, Issue. 6, pp. 829-836, 2001.

(10) Tzu-chao Lin and Pao-Ta Yu : "Salt-Pepper Impulse Noise Detection and Removal Using Multiple Thresholds for Image Restoration", Journal of Information Science and Engineering 22, pp. 189-198, 2006.

(11) Bae-Muu Chang, Hung-Hsu Tsai, Xuan-Ping Lin, and Pao-Ta Yu : "Design of Median-type Filters with an Impulse Noise Detector Using Decision Tree and Particle Swarm Optimization for Image Restoration", ComSIS, Vol. 7, No. 4, pp. 859- 882, 2010.

(12) Arumugam. R, Vellingiri. K, Habeebrakuman.WF and Mohan. K : "A noise denoising approach for the removal of impulse noise from color images and video sequences", Journal of Image Anal Stereol 31, pp. 185-191, 2012.

(13) Jun-Seon. Kim and Hyun Wook Park : "Adaptive 3-D median filtering for restoration of an image sequence corrupted by impulse noise", Image Communication Vol.16, Issue.7, pp. 657- 668, 2001.

(14) Hamid R Tizhoosh : "Image thresholding using Type II Fuzzy Sets", Pattern Recognition 38, pp. 2363-2372, 2005.

(15) Sun Zhong-gui, Chen Jie and Meng Guang-wu : An Impulse Noise Image Filter Using Fuzzy Sets", Elsevier trans of International Symposiums on Information Processing, pp. 183-186, 2008.

(16)Zhou Wang, Alan C. Bovik, Hamid R. Sheikh and Eero P. Simoncelli : "Image Quality Assessment: From Error Visibility to Structural Similarity", IEEE Transactions on image processing, Vol. 13, No. 4, pp. 600-612, 2004. 